\title{
Quivers and the Euclidean algebra (Extended abstract)
}

\author{
Alistair Savage $\|^{\dagger}$ \\ University of Ottawa, Ottawa, Ontario, Canada
}

\begin{abstract}
We show that the category of representations of the Euclidean group $E(2)$ is equivalent to the category of representations of the preprojective algebra of the quiver of type $A_{\infty}$. Furthermore, we consider the moduli space of $E(2)$-modules along with a set of generators. We show that these moduli spaces are quiver varieties of the type considered by Nakajima. These identifications allow us to draw on known results about preprojective algebras and quiver varieties to prove various statements about representations of $E(2)$. In particular, we show that $E(2)$ has wild representation type but that if we impose certain combinatorial restrictions on the weight decompositions of a representation, we obtain only a finite number of indecomposable representations.

Résumé. Nous montrons que la catégorie des représentations du groupe d'Euclide $E(2)$ est équivalente à la catégorie des représentations de l'algèbre préprojective de type $A_{\infty}$. De plus, nous considérons l'espace classifiant de modules de $E(2)$ avec un ensemble de générateurs. Nous montrons que ces espaces sont de variétés de carquois de Nakajima. Cette identification nous permet d'utiliser des résultats des algèbres préprojectives et des variétés de carquois pour prouver des affirmations sur des représentations de $E(2)$. En particulier, nous montrons que le type de répresentations de $E(2)$ est sauvage mais si nous imposons des restrictions aux poids d'une représentation, il y a seulement un nombre fini de représentations qui ne sont pas décomposables.
\end{abstract}

Keywords: Quiver, quiver variety, directed graph, Euclidean algebra, Euclidean group, preprojective algebra

\section{Introduction}

The Euclidean group $E(n)=\mathbb{R}^{n} \rtimes S O(n)$ is the group of isometries of $n$-dimensional Euclidean space. The study of these objects, at least in cases $n=2,3$, predates even the concept of a group. In this paper we will focus on the Euclidean group $E(2)$. Even in this case, much is still unknown about the representation theory.

All finite-dimensional irreducible unitary representations of $E(2)$ are one-dimensional. The infinitedimensional unitary irreducible representations have received considerable attention (see [1, 2, 3]). There also exist finite-dimensional nonunitary indecomposable representations and much less is known about these. However, they play an important role in mathematical physics and the representation theory of the Poincaré group. The group E(2) also appears in the Chern-Simons formulation of Einstein gravity in

\footnotetext{
${ }^{\dagger}$ This research was supported in part by the Natural Sciences and Engineering Research Council (NSERC) of Canada 1365-8050 @ 2008 Discrete Mathematics and Theoretical Computer Science (DMTCS), Nancy, France
} 
$2+1$ dimensions. In the case when the space-time has Euclidean signature and the cosmological constant vanishes, the phase space of gravity is the moduli space of flat $E(2)$-connections.

In the current paper, we relate the representation theory of the Euclidean group $E(2)$ to the representation theory of preprojective algebras of quivers of type $A_{\infty}$. In fact, we show that the categories of representations of the two are equivalent. Furthermore, we consider the moduli space of representations of $E(2)$ along with a set of generators. We show that these moduli spaces are quiver varieties of the type considered by Nakajima in [12, 13]. These identifications allow us to draw on known results about preprojective algebras and quiver varieties to prove various statements about representations of $E(2)$. In particular, we show that the Lie algebra of $E(2)$ is of wild representation type but that if we impose certain restrictions on the weight decomposition of a representation, we obtain only a finite number of indecomposable representations.

\section{The Euclidean algebra}

Let $E(2)=\mathbb{R}^{2} \rtimes S O(2)$ be the Euclidean group of motions in the plane and let $\mathfrak{e}(2)$ be the complexification of its Lie algebra. We call $\mathfrak{e}(2)$ the (three-dimensional) Euclidean algebra. It has basis $\left\{p_{+}, p_{-}, l\right\}$ and commutation relations

$$
\left[p_{+}, p_{-}\right]=0, \quad\left[l, p_{ \pm}\right]= \pm p_{ \pm} .
$$

Since $S O(2)$ is compact, the category of finite-dimensional $E(2)$-modules is equivalent to the category of finite dimensional $\mathfrak{e}(2)$-modules in which $l$ acts semisimply with integer eigenvalues. Will will use the term $\mathfrak{e}(2)$-module to refer only to such modules. For $k \in \mathbb{Z}$, we shall write $V_{k}$ to indicate the eigenspace of $l$ with eigenvalue $k$ (the $k$-weight space). Thus, for an $\mathfrak{e}(2)$-module $V$, we have the weight space decomposition

$$
V=\bigoplus_{k} V_{k}, \quad V_{k}=\{v \in V \mid l \cdot v=k v\}, \quad k \in \mathbb{Z}
$$

and

$$
p_{+} V_{k} \subseteq V_{k+1}, \quad p_{-} \subseteq V_{k-1} .
$$

We may form the tensor product of any representation $V$ with the character $\chi_{n}$ for $n \in \mathbb{Z}$. Here $\chi_{n}$ is the one-dimensional module $\mathbb{C}$ on which $p_{ \pm}$act by zero and $l$ acts by multiplication by $n$. Then a weight space $V_{k}$ of weight $k$ becomes a weight space $V_{k} \otimes \chi_{n}$ of weight $k+n$. In this way, we may "shift weights" as we please.

For $k \in \mathbb{Z}$, let $\mathbf{e}^{k}$ be the element of $\left(\mathbb{Z}_{\geq 0}\right)^{\mathbb{Z}}$ with $k$ th component equal to one and all others equal to zero. For an $\mathfrak{e}(2)$-module $V$ we define

$$
\operatorname{dim} V=\sum_{k \in \mathbb{Z}}\left(\operatorname{dim} V_{k}\right) \mathbf{e}^{k}
$$

\section{Preprojective algebras}

For $a, b \in \mathbb{Z}$ with $a \leq b$, let $Q_{a, b}$ be the quiver with vertex set $I=\{k \in \mathbb{Z} \mid a \leq k \leq b\}$ and arrows $H=\left\{h_{i} \mid a \leq i \leq b-1\right\}$ with out $\left(h_{i}\right)=i$ and $\operatorname{in}\left(h_{i}\right)=i+1$. The quiver $Q_{\infty}$ has vertex set $I=\mathbb{Z}$ and arrows $H=\left\{h_{i} \mid i \in \mathbb{Z}\right\}$ with out $\left(h_{i}\right)=i$ and in $\left(h_{i}\right)=i+1$. 
Let $Q=\left(I, H\right.$, out, in) be a quiver without loops and let $Q^{*}=\left(I, H^{*}\right.$, out*, in $\left.{ }^{*}\right)$ be the double quiver of $Q$. By definition,

$$
\begin{aligned}
& H^{*}=\{h \mid h \in H\} \cup\{\bar{h} \mid h \in H\}, \\
\text { out }^{*}(h)=\operatorname{out}(h), \quad & \operatorname{in}^{*}(h)=\operatorname{in}(h), \quad \text { out }^{*}(\bar{h})=\operatorname{in}(h), \quad \operatorname{in}(\bar{h})=\operatorname{out}(h) .
\end{aligned}
$$

From now on, we will write in and out for in* and out ${ }^{*}$ respectively. Since in* ${ }_{H}=$ in and out* $\left.\right|_{H}=$ out, this should cause no confusion.

For $i \in I$ let

$$
r_{i}=\sum_{h \in H, \text { out }(h)=i} \bar{h} h-\sum_{h \in H, \operatorname{in}(h)=i} h \bar{h}
$$

be the Gelfand-Ponomarev relation associated to $i$. The preprojective algebra $P(Q)$ corresponding to $Q$ is defined to be

$$
P(Q)=\mathbb{C} Q^{*} / J
$$

where $J$ is the two-sided ideal generated by the relations $r_{i}$ for $i \in I$.

Let $\mathcal{V}(I)$ denote the category of finite-dimensional $I$-graded vector spaces with morphisms being linear maps respecting the grading. A representation of the quiver $Q^{*}$ is an element $\mathbf{V} \in \mathcal{V}(I)$ along with a linear map $x_{h}: V_{\text {out }(h)} \rightarrow V_{\operatorname{in}(h)}$ for each $h \in H^{*}$. We let

$$
\operatorname{rep}\left(Q^{*}, V\right)=\bigoplus_{h \in H^{*}} \operatorname{Hom}_{\mathbb{C}}\left(V_{\text {out }(h)}, V_{\mathrm{in}(h)}\right)
$$

be the affine variety consisting of representations of $Q^{*}$ with underlying vector space $\mathbf{V}$. A representation of a quiver can be naturally interpreted as a $\mathbb{C} Q^{*}$-module structure on $\mathbf{V}$. For a path $p=h_{n} h_{n-1} \ldots h_{1}$ in $Q^{*}$, we let

$$
x_{p}=x_{h_{n}} x_{h_{n-1}} \cdots x_{h_{1}} .
$$

We say a representation $x \in \operatorname{rep}\left(Q^{*}, V\right)$ satisfies the relation $\sum_{j=1}^{k} a_{j} p_{j}$, if

$$
\sum_{j=1}^{k} a_{j} x_{p_{j}}=0 .
$$

If $R$ is a set of relations, we denote by $\operatorname{rep}\left(Q^{*}, R, \mathbf{V}\right)$ the set of all representations in $\operatorname{rep}\left(Q^{*}, \mathbf{V}\right)$ satisfying all relations in $R$. This is a closed subvariety of $\operatorname{rep}\left(Q^{*}, \mathbf{V}\right)$. Every element of $\operatorname{rep}\left(Q^{*}, J, \mathbf{V}\right)$ can be naturally interpreted as a $P(Q)$-module structure on $\mathbf{V}$ and so we also write

$$
\bmod (P(Q), \mathbf{V})=\operatorname{rep}(Q, J, \mathbf{V})
$$

for the affine variety of $P(Q)$-modules with underlying vector space $\mathbf{V}$.

The algebraic group $G_{\mathbf{V}}=\prod_{i \in I} G L\left(V_{i}\right)$ acts on $\bmod (P(Q), \mathbf{V})$ by

$$
g \cdot x=\left(g_{i}\right) \cdot\left(x_{h}\right)=\left(g_{\mathrm{in}(h)} x_{h} g_{\mathrm{out}(h)}^{-1}\right)_{h} .
$$


Two $P(Q)$-modules are isomorphic if and only if they lie in the same orbit. For a dimension vector $\mathbf{v} \in\left(Z_{\geq 0}\right)^{I}$, let

$$
\mathbf{V}^{\mathbf{v}}=\bigoplus_{i \in I} \mathbb{C}^{\mathbf{v}_{i}}, \quad \bmod (P(Q), \mathbf{v})=\bmod \left(P(Q), \mathbf{V}^{\mathbf{v}}\right), \quad G_{\mathbf{v}}=G_{\mathbf{V}^{\mathbf{v}}}
$$

Then we have that $\bmod (P(Q), \mathbf{V}) \cong \bmod (P(Q), \operatorname{dim} \mathbf{V})$ for all $\mathbf{V} \in \mathcal{V}(I)$. Therefore, we will blur the distinction between $\bmod (P(Q), \mathbf{V})$ and $\bmod (P(Q), \operatorname{dim} \mathbf{V})$.

We say an element $x \in \bmod (P(Q), \mathbf{V})$ is nilpotent if there exists an $N \in \mathbb{Z}_{>0}$ such that for any path $p$ of length greater than $N$, we have $x_{p}=0$. Denote the closed subset of nilpotent elements of $\bmod (P(Q), \mathbf{V})$ by $\Lambda_{\mathbf{V}, Q}$ and let $\Lambda_{\mathbf{v}, Q}=\Lambda_{\mathbf{V} v, Q}$. The varieties $\Lambda_{\mathbf{V}, Q}$ are called nilpotent varieties or Lusztig quiver varieties. Lusztig [11] has shown that the $\Lambda_{\mathbf{V}, Q}$ have pure $\operatorname{dimesion} \operatorname{dim}(\operatorname{rep}(Q, \mathbf{V}))$ and that their irreducible components are in one-to-one correspondence with a basis of a certain weight space of the lower half of the enveloping algebra of $\mathfrak{g}$, the Kac-Moody algebra whose Dynkin graph is the underlying graph of $Q$.

Proposition 3.1 For a quiver $Q$, the following are equivalent:

1. $P(Q)$ is finite-dimensional,

2. $\Lambda_{\mathbf{V}, Q}=\bmod (P(Q), \mathbf{V})$ for all $\mathbf{V}=\mathcal{V}(I)$,

3. $Q$ is a Dynkin quiver (i.e. its underlying graph is of $A D E$ type).

Proof: The equivalence of (1) and (3) is well-known (see for example [14]). That (2) implies (3) was proven by Crawley-Boevey [4] and the converse was proven by Lusztig [11, 14.2].

Thus, for a Dynkin quiver $Q$, nilpotency holds automatically and $\Lambda_{\mathbf{V}, Q}$ is just the variety of representations of the preprojective algebra $P(Q)$ with underlying vector space $\mathbf{V}$.

The representation type of the preprojective algebras is known.

Proposition 3.2 ([5, 9]) Let $Q$ be a finite quiver. Then the following hold:

1. $P(Q)$ is of finite representation type if and only if $Q$ is of Dynkin type $A_{n}, n \leq 4$,

2. $P(Q)$ is of tame representation type if and only if $Q$ is of Dynkin type $A_{5}$ or $D_{4}$.

Thus $P(Q)$ is of wild representation type if $Q$ is not of Dynkin type $A_{n}, n \leq 5$, or $D_{4}$.

In the sequel, we will refer to the preprojective algebra $P\left(Q_{\infty}\right)$. While $Q_{\infty}$ is not a finite quiver, any finite-dimensional representation is supported on finitely many vertices and thus is a representation of a quiver of type $A_{n}$ for sufficiently large $n$. Thus we deduce the following.

Corollary 3.3 All finite-dimensional representations of $Q_{\infty}$ are nilpotent and $P\left(Q_{\infty}\right)$ is of wild representation type. 


\section{Representations of the Euclidean algebra and preprojective al- gebras}

In this section we examine the close relationship between representations of the Euclidean algebra $\mathfrak{e}(2)$ and the preprojective algebras of type $A_{n}$. Let $\operatorname{Mod} \mathfrak{e}(2)$ be the category of $\mathfrak{e}(2)$-modules. For $a \leq b$, let $\operatorname{Mod}_{a, b} \mathfrak{e}(2)$ be the full subcategory consisting of representations $V$ such that $V_{k}=0$ for $k<a$ or $k>b$. Then $\operatorname{Mod} \mathfrak{e}(2)$ is the limit of the categories $\operatorname{Mod}_{a, b} \mathfrak{e}(2)$. For $\mathbf{v} \in\left(\mathbb{Z}_{\geq 0}\right)^{\mathbb{Z}}$, we also define $\operatorname{Mod}_{a, b}^{\mathbf{v}} \mathfrak{e}(2)$ to be the full subcategory of $\operatorname{Mod}_{a, b} \mathfrak{e}(2)$ consisting of representations $V$ such that $\operatorname{dim} V=\mathbf{v}$.

Let $\operatorname{Mod} P(Q)$ be the category of finite-dimensional $P(Q)$-modules and for $\mathbf{v} \in\left(\mathbb{Z}_{\geq 0}\right)^{I}$, let $\mathbf{M o d}^{\mathbf{v}} P(Q)$ be the full subcategory consisting of modules of graded dimension $\mathbf{v}$.

Proposition 4.1 We have the following equivalences of categories.

1. $\operatorname{Mod}_{a, b}^{\mathbf{v}} \mathfrak{e}(2) \cong \operatorname{Mod}^{\mathbf{v}} P\left(Q_{a, b}\right), \operatorname{Mod}_{a, b} \mathfrak{e}(2) \cong \operatorname{Mod} P\left(Q_{a, b}\right)$,

2. $\operatorname{Mod}^{\mathbf{v}} \mathfrak{e}(2) \cong \operatorname{Mod}^{\mathbf{v}} P\left(Q_{\infty}\right) \operatorname{Mod} \mathfrak{e}(2) \cong \operatorname{Mod} P\left(Q_{\infty}\right)$.

Theorem 4.2 The following statements hold.

1. The Euclidean algebra $\mathfrak{e}(2)$ has wild representation type, and

2. for $a, b \in \mathbb{Z}$ with $0 \leq b-a \leq 3$, there are a finite number isomorphism classes of indecomposable $\mathfrak{e}(2)$-modules $V$ whose weights lie between $a$ and $b$; that is, such that $V_{k}=0$ for $k<a$ or $k>b$.

Corollary 4.3 Let $A$ be a finite subset of $\mathbb{Z}$ with the property that $A$ does not contain any five consecutive integers. Then there are a finite number of isomorphism classes of indecomposable $\mathfrak{e}(2)$-modules $V$ with the property that $V_{k}=0$ if $k \notin A$.

Douglas [7] has shown that there are finitely many indecomposable $\mathfrak{e}(2)$-modules (up to isomorphism) of dimensions five and six. Corollary 4.3 can be used to simply such proofs by allowing one to restrict attention to modules with certain weight space dimensions. We also point out that the graphs appearing in [7] roughly correspond, under the equivalence of categories in Proposition 4.1, to the diagrams appearing in the enumeration of irreducible components of quiver varieties given in [8].

\section{Nakajima quiver varieties}

Let $Q$ be the quiver $Q_{\infty}$ or $Q_{a, b}$ for some $a \leq b$. For $\mathbf{V}, \mathbf{W} \in \mathcal{V}(I)$ define

$$
L_{Q}(\mathbf{V}, \mathbf{W})=\Lambda_{\mathbf{V}, Q} \oplus \bigoplus_{i \in I} \operatorname{Hom}_{\mathbb{C}}\left(W_{i}, V_{i}\right)
$$

We denote points of $L_{Q}(\mathbf{V}, \mathbf{W})$ by $(x, s)$ where $x=\left(x_{h}\right)_{h \in H^{*}} \in \Lambda_{\mathbf{V}, Q}$ and $s=\left(s_{i}\right)_{i \in I} \in \operatorname{Hom}_{\mathbb{C}}\left(W_{i}, V_{i}\right)$. We say an $I$-graded subspace $\mathbf{U}$ of $\mathbf{V}$ is $x$-invariant if $x_{h}\left(U_{\text {out }(h)}\right) \subseteq U_{\text {in }(h)}$ for all $h \in H^{*}$. We say a point $(x, s) \in L_{Q}(\mathbf{V}, \mathbf{W})$ is stable if the following property holds: If $\mathbf{U}$ is an $I$-graded $x$-invariant subspace of $\mathbf{V}$ containing im $s$, then $\mathbf{U}=\mathbf{V}$. We denote by $L_{Q}(\mathbf{V}, \mathbf{W})^{\text {st }}$ the set of stable points.

The group $G_{\mathbf{V}}$ acts on $L_{Q}(\mathbf{V}, \mathbf{W})$ by

$$
g \cdot(x, s)=\left(g_{i}\right)_{i \in I} \cdot\left(\left(x_{h}\right)_{h \in H^{*}},\left(s_{i}\right)_{i \in I}\right)=\left(\left(g_{\text {in }(h)} x_{h} g_{\text {out }(h)}^{-1}\right)_{h \in H^{*}},\left(g_{i} s_{i}\right)_{i \in I}\right) .
$$


The action of $G_{\mathbf{V}}$ preserves the stability condition and the stabilizer in $G_{\mathbf{V}}$ of a stable point is trivial. We form the quotient

$$
\mathcal{L}_{Q}(\mathbf{V}, \mathbf{W})=L_{Q}(\mathbf{V}, \mathbf{W})^{\mathrm{st}} / G_{\mathbf{V}}
$$

The $\mathcal{L}_{Q}(\mathbf{V}, \mathbf{W})$ are called Nakajima quiver varieties. For $\mathbf{v}, \mathbf{w} \in\left(\mathbb{Z}_{\geq 0}\right)^{I}$, we set

$$
L_{Q}(\mathbf{v}, \mathbf{w})=L_{Q}\left(\mathbf{V}^{\mathbf{v}}, \mathbf{V}^{\mathbf{w}}\right), \quad L_{Q}(\mathbf{v}, \mathbf{w})^{\mathrm{st}}=L_{Q}\left(\mathbf{V}^{\mathbf{v}}, \mathbf{V}^{\mathbf{w}}\right)^{\mathrm{st}}, \quad \mathcal{L}_{Q}(\mathbf{v}, \mathbf{w})=\mathcal{L}_{Q}\left(\mathbf{V}^{\mathbf{v}}, \mathbf{V}^{\mathbf{w}}\right) .
$$

We then have

$$
\begin{gathered}
L_{Q}(\mathbf{V}, \mathbf{W}) \cong L_{Q}(\operatorname{dim} \mathbf{V}, \operatorname{dim} \mathbf{W}), \quad L_{Q}(\mathbf{V}, \mathbf{W})^{\mathrm{st}} \cong L_{Q}(\operatorname{dim} \mathbf{V}, \operatorname{dim} \mathbf{W})^{\mathrm{st}}, \\
\mathcal{L}_{Q}(\mathbf{V}, \mathbf{W}) \cong \mathcal{L}_{Q}(\operatorname{dim} \mathbf{V}, \operatorname{dim} \mathbf{W}),
\end{gathered}
$$

and so we often blur the distinction between these pairs of isomorphic varieties.

\section{Moduli spaces of representations of the Euclidean algebra}

Given that $\mathfrak{e}(2)$ has wild representation type, it is prudent to restrict one's attention to certain subclasses of modules and to attempt a classification of the modules belonging to these classes. One possible approach is to impose a restriction on the number of generators of a representation (see [6, 7]). In this section we will examine the relationship between moduli spaces of representations of the Euclidean algebra along with a set of generating vectors and Nakajima quiver varieties.

Let $V$ be a finite-dimensional $\mathfrak{e}(2)$-module. For $u_{1}, u_{2}, \ldots, u_{n} \in V$, we denote by $\left\langle u_{1}, \ldots, u_{n}\right\rangle$ the submodule of $V$ generated by $\left\{u_{1}, \ldots, u_{n}\right\}$. It is defined to be the smallest submodule of $V$ containing all the $u_{i}$. A element $u \in \mathbf{V}$ is called a weight vector if it lies in some weight space $V_{k}$ of $V$. For a weight vector $u$, we let wt $u=k$ where $u \in V_{k}$. We say that $\left\{u_{1}, \ldots, u_{n}\right\}$ is a set of generators of $V$ if each $u_{i}$ is a weight vector and $\left\langle u_{1}, \ldots, u_{n}\right\rangle=V$. For $\mathbf{v} \in\left(\mathbb{Z}_{\geq 0}\right)^{\mathbb{Z}}$, we let $|\mathbf{v}|=\sum_{k \in \mathbb{Z}} v_{k}$.

Definition 6.1 For $\mathbf{v}, \mathbf{w} \in\left(\mathbb{Z}_{\geq 0}\right)^{\mathbb{Z}}$, let $E(\mathbf{v}, \mathbf{w})$ be the set of all

$$
\left(V,\left(u_{k}^{j}\right)_{k \in \mathbb{Z}, 1 \leq j \leq \mathbf{w}_{k}}\right)
$$

where $V$ is a finite-dimensional $\mathfrak{e}(2)$-module with $\operatorname{dim} V=\mathbf{v}$ and $\left(u_{k}^{j}\right)_{k \in \mathbb{Z}, 1 \leq j \leq \mathbf{w}_{k}}$ is a set of generators of $V$ such that $\mathrm{wt} u_{k}^{j}=\mathbf{e}^{k}$. We say that two elements $\left(V,\left(u_{k}^{j}\right)\right)$ and $\left(\tilde{V},\left(\tilde{u}_{k}^{j}\right)\right)$ of $E(\mathbf{v}, \mathbf{w})$ are equivalent if there exists a $\mathfrak{e}(2)$-module isomorphism $\phi: V \rightarrow \tilde{V}$ such that $\phi\left(u_{k}^{j}\right)=\tilde{u}_{k}^{j}$. We denote the set of equivalence classes by $\mathcal{E}(\mathbf{v}, \mathbf{w})$.

Theorem 6.2 There is a natural one-to-one correspondence between $\mathcal{E}(\mathbf{v}, \mathbf{w})$ and $\mathcal{L}_{Q^{\infty}}(\mathbf{v}, \mathbf{w})$.

A partition is a sequence of non-increasing natural numbers $\lambda=\left(\lambda_{1}, \lambda_{2}, \ldots, \lambda_{l}\right)$. The corresponding Young diagram is a collection of rows of square boxes which are left justified, with $\lambda_{i}$ boxes in the $i$ th row, $1 \leq i \leq l$. We will identify a partition and its Young diagram and we denote by $\mathcal{Y}$ the set of all partitions (or Young diagrams). If $b$ is a box in a Young diagram $\lambda$, we write $x \in \lambda$ and we denote the box in the $i$ th column and $j$ th row of $\lambda$ by $x_{i, j}$ (if such a box exists). The residue of $x_{i, j} \in \lambda$ is defined to be res $x_{i, j}=i-j$. For $\lambda \in \mathcal{Y}$ and $a \in \mathbb{Z}$, define $\mathbf{v}^{\lambda, a} \in\left(\mathbb{Z}_{\geq 0}\right)^{\mathbb{Z}}$ by setting $\mathbf{v}_{i+a}^{\lambda, a}$ to be the number of boxes in $\lambda$ of residue $i$. 
Proposition 6.3 For $\lambda \in \mathcal{Y}$, there exists a unique $\mathfrak{e}(2)$-module $V$ (up to isomorphism) with a single generator of weight $a \in \mathbb{Z}$ and $\operatorname{dim} V=\mathbf{v}^{\lambda, a}$. It is given by

$$
\begin{aligned}
V & =\operatorname{Span}_{\mathbb{C}}\{x \mid x \in \lambda\} \\
l\left(x_{i, j}\right) & =\operatorname{res} x_{i, j}=a+i-j \\
p_{+}\left(x_{i, j}\right) & =x_{i+1, j} \\
p_{-}\left(x_{i, j}\right) & =x_{i, j+1},
\end{aligned}
$$

where we set $x_{i, j}=0$ if there is no box of $\lambda$ in the ith column and $j$ th row.

For $\mathbf{v} \in\left(\mathbb{Z}_{\geq 0}\right)^{\mathbb{Z}}$ such that $\mathbf{v} \neq \mathbf{v}^{\lambda, a}$ for all $\lambda \in \mathcal{Y}$ and $a \in \mathbb{Z}$, there are no $\mathfrak{e}(2)$-modules $V$ with a single generator and $\operatorname{dim} V=\mathbf{v}^{\lambda, a}$

Thus $\mathfrak{e}(2)$-modules with a single generator of a fixed weight are determined completely by the dimensions of their weight spaces. This was proven directly by Gruber and Henneberger in [10]. However, our knowledge of the precise relationship between quivers and the Euclidean algebra allows us to use known results about quivers and quiver varieties to simplify such proofs.

\section{References}

[1] H. Ahmedov and I. H. Duru. Unitary representations of the two-dimensional Euclidean group in the Heisenberg algebra. J. Phys. A, 33(23):4277-4281, 2000.

[2] K. Baumann. Vector and ray representations of the Euclidean group E(2). Rep. Math. Phys., 34(2):171-180, 1994.

[3] A. M. Boyarskiı̌ and T. V. Skrypnik. Singular orbits of a coadjoint representation of Euclidean groups. Uspekhi Mat. Nauk, 55(3(333)):169-170, 2000.

[4] W. Crawley-Boevey. Geometry of the moment map for representations of quivers. Compositio Math., 126(3):257-293, 2001.

[5] V. Dlab and C. M. Ringel. The module theoretical approach to quasi-hereditary algebras. In Representations of algebras and related topics (Kyoto, 1990), volume 168 of London Math. Soc. Lecture Note Ser, pages 200-224. Cambridge Univ. Press, Cambridge, 1992.

[6] A. Douglas. A classification of the finite dimensional, indecomposable representations of the Euclidean algebra $\mathfrak{e}(2)$ having two generators. PhD thesis, University of Toronto, 2006.

[7] A. Douglas. Finite dimensional representations of the Euclidean algebra $\mathfrak{e}(2)$ having two generators. J. Math. Phys., 47(5):053506, 14, 2006.

[8] I. B. Frenkel and A. Savage. Bases of representations of type $A$ affine Lie algebras via quiver varieties and statistical mechanics. Int. Math. Res. Not., (28):1521-1547, 2003.

[9] C. Geiss and J. Schröer. Varieties of modules over tubular algebras. Colloq. Math., 95(2):163-183, 2003. 
[10] B. Gruber and W. C. Henneberger. Representations of the Euclidean group in the plane. Nuovo Cimento B (11), 77(2):203-233, 1983.

[11] G. Lusztig. Quivers, perverse sheaves, and quantized enveloping algebras. J. Amer. Math. Soc., 4(2):365-421, 1991.

[12] H. Nakajima. Instantons on ALE spaces, quiver varieties, and Kac-Moody algebras. Duke Math. J., 76(2):365-416, 1994.

[13] H. Nakajima. Quiver varieties and Kac-Moody algebras. Duke Math. J., 91(3):515-560, 1998.

[14] I. Reiten. Dynkin diagrams and the representation theory of algebras. Notices Amer. Math. Soc., 44(5):546-556, 1997. 